\title{
Application and experimental study of reinforcement method of pasting steel plates on $\mathrm{RC}$ arch bridges
}

\author{
Zhanbiao Zhang $^{\mathrm{a}}{ }^{*}$, Qian Wang ${ }^{\mathrm{b}}$ and Cailiang Huang ${ }^{\mathrm{c}}$ \\ Dalian University of Technology, China \\ azzbiao@mail.dlut.edu.cn, ${ }^{\mathrm{b}}$ wang_qian_82@163.com, ${ }^{\mathrm{c}}$ cailiang@dlut.edu.cn
}

\begin{abstract}
Keywords: reinforced concrete arch bridge; pasting steel plate; reinforcement method; anchor bolt; destructive loading test

Abstract. The Shengli Bridge is a five-span continuous arch bridge constructed by Japanese 108 years ago in Dalian, China. Lacking of maintenance and repair, lots of longitudinal and transversal cracks are found on the bottom surfaces of arch ribs, as a consequence, steel bars are exposed and corroded extensively. Steel pasting method is adopted to enhance the structural bearing capacity and service performance after comprehensive comparison with other common repair methods for arch bridges. To validate and optimize the strengthening effect, and to look for a feasible construction technology applicable for reinforcement project of real bridge, destructive loading test was took on four reinforced concrete arch models, one of which without any reinforcement while other three strengthened by different measures including bonding steel plate, bonding steel plate combined with anchor bolts, and bonding I-steel plate, respectively. Results showed that cracking load of structures was effectively increased by each reinforcement measure; failure characteristic was remarkably improved with the existence of anchor bolts; while bearing capacity cannot be highly developed by merely bonding I-steel plate.
\end{abstract}

\section{Introduction}

The Shengli Bridge in Dalian is a five-span continuous arch bridge over the railways constructed by Japanese in 1907 . Total length of bridge is $94.82 \mathrm{~m}$, with three main spans of $26.44 \mathrm{~m}, 25.34 \mathrm{~m}, 26.44 \mathrm{~m}$ long respectively, the middle span with a rise-span ratio of 1/6. Because of the long history and lacking of maintenance, arch ring and subsidiary facilities are seriously damaged. Lots of longitudinal cracks emerged along the construction joints, leading to the integrated arch ring broken up into 9 mutually independent ribs, in addition, transversal cracks are also found around each mid-span. The existence of these cracks and failure of waterproof and drainage system of upper structures leaving the main arch rings undergo frequent erosion of rainwater, large scale of seepage, whitening, concrete shedding and even stalactites can be seen on the bottom surface of arch ribs, let the steel bars exposed to air and corroded extensively. According to detection, Shengli Bridge is a dangerous bridge with an integrate grade of $\mathrm{E}$ and need to be strengthened for safety.

Each common reinforcement method for arch bridge, such as section enlargement method, external prestressing method, external bonding method, and transform structural system method, have their own advantages and disadvantages and limit scope of applicability. Shengli Bridge located at urban district with busy railway across below it, only $20 \mathrm{~cm}$ height is left from railway clearance to bottom of the arch ring. Thus, when choosing a final reinforcement method, not only mechanical behavior, utmost care should also be taken to the factors of safety, space, technique, costs and construction period. Finally, bonding steel method is adopted as a proposal reinforcement method for Shengli Bridge through comprehensive comparison with other strengthening method.

Many studies have been developed on reinforcement method of pasting steel plates on concrete beams at home and abroad. R. N. Swamy presents comprehensive test data on the first crack load, cracking behavior, structural deformation, serviceable loads, and ultimate strength, of reinforced concrete beams strengthened with steel plates bonded on the tension face in 1987. the result showed that plated beams have enhanced flexural stiffness which control cracking and deformation at all load levels, two tentative design criteria, the plate-width-to-thickness ratio and neutral axis depth, are suggested for plated beams to ensure their full 
flexural capacity and ductility at failure [1]. R. Jones present a simple theoretical study of the force systems at the plate/glue and the glued concrete interfaces in 1988, suggested that high stress concentrations and peeling forces are present at the end of the plates when composite beam is loaded in flexure [2].

Argument appeared before final reinforcement scheme of Shengli Bridge was drawn up, some insist anchor bolts are essential for better cohesion while others not, few argue that I-steel is stronger and of course obtain more remarkable strengthening effect than that of steel plate. This test aims to make clear whether the strengthening plan could improve structural force condition and if it feasible in reinforcement project of real bridge, based on this, analyze and optimize the reinforcement scheme. Specific aspects include: whether the glue used in real bridge is reliable, is it necessary to set anchor bolts between the steel plate and concrete, compare the strengthening effect of bonding steel plate with that of bonding I-steel. Besides, on the basis of the drafted reinforcement technology of arch models, conclude and analyze problems across the strengthening process, finally obtain a rational, safe and feasible reinforcement technology for Shengli Bridge.

\section{Experiment Design.}

\section{Design of Models}

Concrete used in the model has a cubic compressive strength of $41.64 \mathrm{MPa}$, steel bar with an yield strength of $420 \mathrm{MPa}$ and an ultimate strength of $595 \mathrm{MPa}$.

Models features are as follows: calculated span of $3000 \mathrm{~mm}$; cross section of $250 \mathrm{~mm} \times 150 \mathrm{~mm}$, represents width and depth, respectively; rise-span ratio of 1/6, which is correspond with real bridge; 3 steel bars with diameter of $10 \mathrm{~mm}$ are distributed to both the upside and downside of the section.

A big concrete arch abutment with large rigidity was designed for each arch model to simulate the hingeless type of real bridge, as shown in Fig. 1. Steel plates were pre-placed in the junction of arch ribs and abutments, which got welded together after ribs were bonded to eliminate rotation. Abutment was prestressed by strands to eliminate longitudinal displacement.
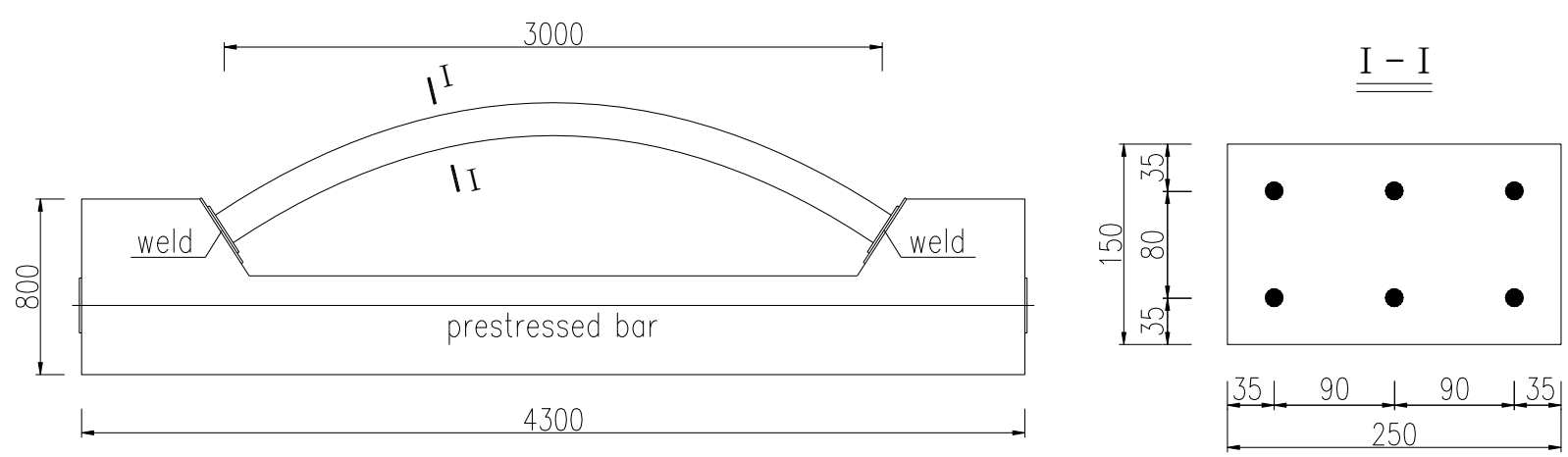

Fig. 1. Layout and reinforcement of models (unit: $\mathrm{mm}$ )

\section{Reinforcement Scheme}

As shown in Fig. 2, Four arch models are made to compare different strengthening effect of each reinforcement measure, one of which without any reinforcement (numbered A) and three other strengthened by different measures, including bonding steel plate (numbered B), bonding steel plate combined with anchor bolts (numbered C) and bonding I-steel plate (numbered D). Each steel plate has a thickness of 3mm and anchor bolt has a diameter of $6 \mathrm{~mm}$, the glue of JGN type, developed by Dalian Institute of Chemical Physics, Chinese Academy of Sciences, has a shear strength of 2.2GPa. 

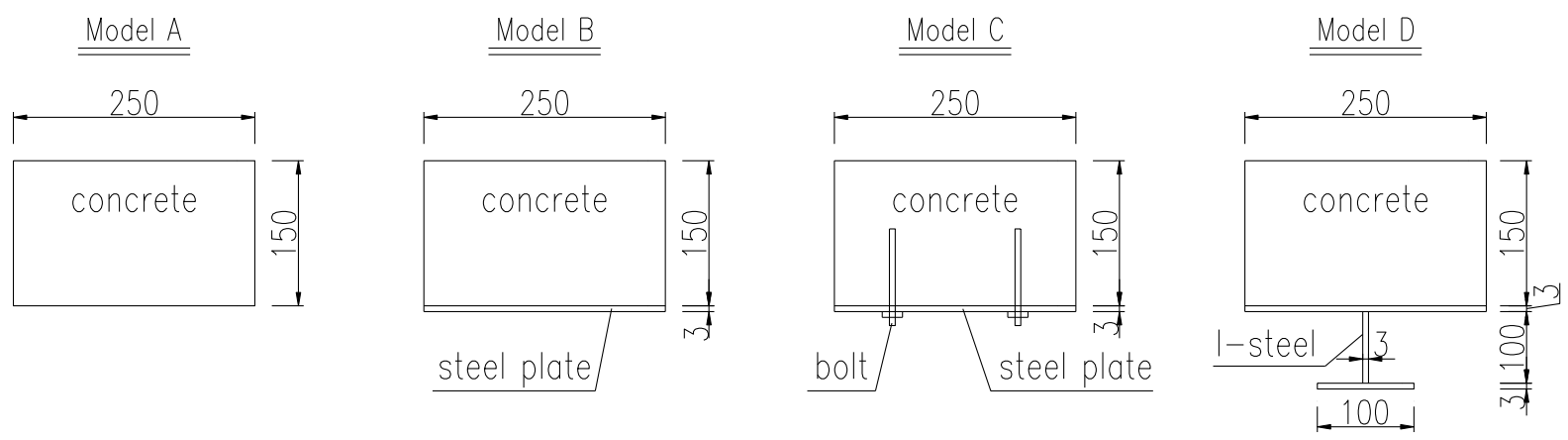

Fig. 2. Layout of strengthening scheme (unit: $\mathrm{mm}$ )

Models are strengthened as the following procedure: a. chisel off cement mortar on concrete surface with a depth about $7 \mathrm{~mm}$ so that stone could be exposed, blow away the dust and clean up the surface with acetone; b. use grinder to removal rust of steel plate until it turns rough and metallic luster; c. mark positions of anchor bolts on concrete bottom surface, at which bore several holes and clean up, fill them with glue and insert anchor bolts, then cure it under appropriate condition; d. punch the steel plate at the corresponding position, install the steel plate to concrete with a $3 \mathrm{~mm}$ thick gasket placed between them. e. close margin of the space but pre-place injection holes, stir the glue according to instructed mixture ratio and infunde it into pressure can, then inject glue to interspace of steel plate and concrete under low pressure. f. cure at room temperature at least 24 hours.

\section{Loading Technique}

A hand jack ranged 50t was used to load at mid-span of each arch model, loading speed was controlled according to a pressure sensor installed between the hand jack and reaction frame. To prevent the local concrete frombeing crushed, a block rubber embedded with thick steel plates was placed upon the center of arch rib. Vertical displacements and critical strains of controlling section are measured by three dial indicator and strain gauges distributed at corresponding location respectively.

\section{Test Results and Discussion.}

Extensive data on the strength, crack, deformation, and failure characteristics, of the four arch models were obtained from tests. Only important results are, however, presented here.

\section{Cracking and Failing Process}

Model A: The first crack appeared on the bottom surface of mid-span section when loading to $120 \mathrm{kN}$, later 1/4 and 3/4 span section began cracking from top surface when load is $190 \mathrm{kN}$. Concrete at upside of mid-span and downside of 1/4 and 3/4 span got crushed near failure, crack at 3/4 span run though the whole oblique section at the same time while the load stopped ascending anymore.

Model B: There was no crack until the steel plate peeled from concrete with a length of $0.4 \mathrm{~m}$ near mid-span when loading to $216 \mathrm{kN}$. It is remarkable, however, that when loading to $356 \mathrm{kN}$, concrete at mid-span smashed in a sudden and the peeling length reached $1.1 \mathrm{~m}$ with glue got sheared.

Model C: Failure process of model C is familiar with that of model B except for a more obvious ductility throughout all load level until failure. Instead of smashed, the section near mid-span got sheared off with a diagonal crack. Besides, length of peeling between steel plate and concrete near failure reduced to about $0.4 \mathrm{~m}$ compared with $1.1 \mathrm{~m}$ measured in model $\mathrm{B}$.

ModelD: Compared with the other three models, modelD obtain a much higher cracking load of $288 \mathrm{kN}$, when the I-steel plate peeling from concrete along the right half span, and several cracks appeared at each controlling section. What's more, when loaded to $290 \mathrm{kN}$, the whole I-steel plate fell down in a sudden and detached from concrete completely, leaving the structure nothing different from model A and failed in flexure at about $320 \mathrm{kN}$ 


\section{Cracking Load and Ultimate Load}

Table 1 shows cracking load and ultimate load of each arch model recorded in the test, relative increase in percentage compared with model A are also presented.

Table 1. Cracking load and ultimate load of each model

\begin{tabular}{ccccc}
\hline Model number & A & B & C & D \\
\hline Cracking load $[\mathrm{kN}]$ & 120 & 216 & 210 & 288 \\
Relative increase [\%] & 0 & 80 & 75 & 140 \\
Ultimate load [kN] & 283 & 356 & 345 & 320 \\
Relative increase [\%] & 0 & 26 & 22 & 13 \\
\hline
\end{tabular}

It is apparent that both three strengthening methods achieve remarkably development in cracking load, and pasting I-steel shows a more favorable effect than pasting steel plate when it comes to the increased range. This is because the steel showed no peeling, thus bear and deformation together, with concrete at low loading level, while the higher stiffness of I-steel contribute a greater flexural rigidity of the composite section than that of steel plate, and delayed the coming of cracks effectively.

However, as load was increasing, so did the deformation, while the stiffness of I-steel plate is great enough to prevent itself from bending together with the concrete and induced generation of a peeling force, which resulting in bonding failure of the glue, finally led to the I-steel entirely detached with the concrete rib in a sudden when load was far less than the bending strength of the composite structure. While familiar phenomenon did not occurred in model $\mathrm{B}$ and model $\mathrm{C}$ for the lower stiffness of $3 \mathrm{~mm}$ steel plate, as mentioned before, the steel plate showed no wholly peeling until failure, so the integrate rigidity was guaranteed, contributing to an effective increase of ultimate strength.

\section{Strain Analysis.}

Longitudinal strains distributed in different depth of each composite section agree with plane-section assumption before the steel plate or I-steel stripped from concrete arch ribs, indicating reliable quality and effectiveness of glue and strengthening technology. Other detailed strains obtained in the test are not presented for limited space.

\section{Conclusion}

Some conclusions can be drawn according to the test: glue and strengthening technology applied in the test guarantee a reliable cohesion between steel plates and concrete arch ribs, and are proposal for reinforcement project of real bridge; cracking load is effectively increased by each strengthening method and bonding I-steel shows the highest; the installation of anchor bolts has no visible contribution on cracking load or ultimate strength, failure characteristic, however, is remarkably improved with the existence of anchor bolts; as for I-steel plate, it is difficult to deform together with concrete for its great stiffness, leading to premature bonding failure occurs without the composite structure achieving its full flexural or compressive strength, indicates that significant difference between the new and old structures should be avoided unless reliable combination is ensured by effective bonding or anchor measures; a tentative design of pasting steel plate and anchored with bolts at the same time is suggested to be an advisable reinforcement scheme for Shengli Bridge.

\section{Acknowledgments}

The research described in this paper was financially supported by the science and technology funds of Liaoning Education Department of China [20131021], and the National Natural Science Foundation of China [51308090]. 


\section{References}

[1] R. N. Swamy, R. Jones, J. W. Bloxham, Structural behavior of reinforced concrete beams strengthened by epoxy-bonded steel plates, The Structural Engineer. 65A (1987) 59-68.

[2] R. Jones, R. N. Swamy, A. Charif, Plate separation and anchorage of reinforced concrete beams strengthened by epoxy-bonded steel plates, The Structural Engineer. 66 (1988) 85-94. 